\title{
Assessment of Spinal Cord Injury via Sparse Modeling of Somatosensory Evoked Potential Signals
}

\author{
H. Mir and H. Al-Nashash \\ Department of Electrical Engineering \\ American University of Sharjah, UAE
}

\author{
J. Kortelainen \\ Center for Machine Vision and Signal Analysis \\ University of Oulu, Finland
}

\author{
A. All \\ SINAPSE Institute \\ National University of Singapore
}

\begin{abstract}
The morphological differences between somatosensory evoked potential (SEP) signals from a normal spinal pathway and spinal pathway affected by spinal cord injury (SCI) provide an indication of the degree of SCI. A sparse representation of the fit between these signals is proposed in this paper as an SCI assessment method. The proposed method is tested on actual SEP signals collected from rodents that have been subjected to spinal transection. Results indicate that the proposed method provides a robust measure of the different degrees of SCI resulting from transection of the spinal cord.
\end{abstract}

Keywords-Somatosensory Evoked Potential (SEP), Spinal Cord Injury (SCI)

\section{INTRODUCTION}

The spinal cord is the pathway through which electrical signals between the limbs and the brain must pass. Spinal cord injury (SCI) will thus impair the electrical signals and result in degraded sensory and motor function [1]. The large number of people around the world who are affected by SCI motivates the need to develop novel diagnostic methods. Such methods are helpful in determining the viability of post-SCI therapeutic treatments.

While imaging approaches for diagnosing SCI such as MRI give information about the area of the injury, electrophysiological measurements such as the somatosensory evoked potential (SEP) can provide information about the functional integrity of the spinal cord. The SEP is collected through external electrical stimulation of the median nerve at the wrist or the posterior tibial nerve at the ankle. Prior work [2]-[4], has shown that the morphological similarity between an SEP signal from a healthy spinal pathway and an SEP signal from an injured spinal pathway can be used as a quantitative measure of the level of SCI and provide a complementary assessment to qualitative behavioral based measures such as the Basso, Beattie, and Bresnahan (BBB) test [5].
This paper describes a method that uses a sparse representation of the similarity between SEP signals from a healthy and SCI damaged spinal pathway in order to assess the severity of the injury [6]. The proposed method models the SCI affected SEP signal as a superposition of time-shifted and amplitude scaled replicas of the SEP signal from a healthy spinal pathway. Using experimental data collected from rodents, it is shown that this model can provide a quantitative assessment of the level of SCI.

The remainder of this paper is organized as follows: Section II describes the experimental protocol used to collect SEP signals from rodents. Section III describes the proposed SCI assessment method. Section IV presents the results of applying the method in Section III to the experimental data described in Section II.

\section{PROTOCOL AND DATA COLLECTION}

The cranium of Sprague-Dawley rodents were implanted with four epidural screw electrodes, which were located above the somatosensory cortex of different limbs. Stainless-steel subdermal needle electrodes were used to stimulate the limbs, resulting in the generation of evoked potentials. Recordings were made 510 days after implanting the electrodes. Two types of spinal transection, namely T7/T8 left hemitransection and T8 complete transection, were performed. Posttransection SEP recordings were performed using a Tucker Davis Technologies (TDT) workstation. All recordings were performed using $1.5 \%$ isoflurane.

\section{SCI QUANTIFICATION}

The SEP signal from a healthy spinal pathway will be used as a baseline reference signal and will be represented as $r[n]$. Similarly, the SEP signal from a SCI damaged spinal pathway will be represented as $s[n]$. The deviation of $s[n]$ from $r[n]$ will be modeled using a filter $h[n]$. As such, through the convolution 
operator:

$$
s[n] \approx \mathbf{h}^{T} \mathbf{r}[n]
$$

where

$$
\mathbf{h}=\left[\begin{array}{c}
h[-M] \\
h[-M+1] \\
\vdots \\
h[M]
\end{array}\right], \mathbf{r}[n]=\left[\begin{array}{c}
r[n+M] \\
r[n+M-1] \\
\vdots \\
r[n-M]
\end{array}\right]
$$

and $M$ is the maximum time-shift. To determine the solution for the filter coefficients $h[n]$ which are encapsulated in $\mathbf{h}$, a standard approach is to use the least squares method, through which, for $N$ timesamples, (1) may be recast as:

$$
\underset{\mathbf{h}}{\arg \min } \sum_{n=0}^{N-1}\left\|s[n]-\mathbf{h}^{T} \mathbf{r}[n]\right\|_{2}^{2}
$$

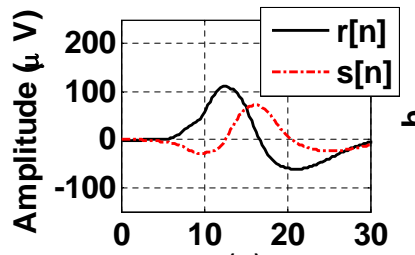

(a)

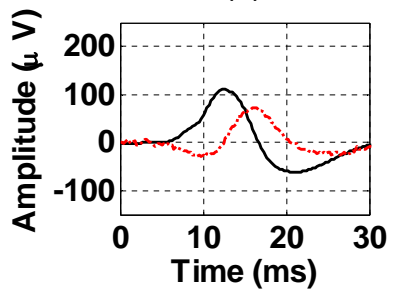

(c)

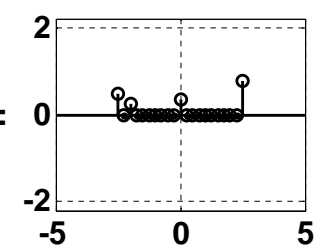

(b)

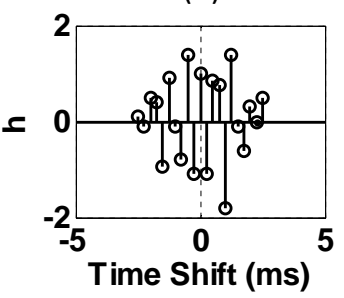

(d)
Figure 1: (a) Sample $r[n]$ and $s[n]$ and (b) resulting least squares fit. (c) Sample $r[n]$ and noisy $s[n]$ and (d) resulting least squares fit.

The least-squares solution, however, is very sensitive to perturbations such as noise and suffers from overfitting. Figure 1(a) shows a sample $r[n]$ and noise-free $s[n]$, while Figure 1(b) shows the resulting fit. Figure 1(c) shows the same sample $r[n]$ as in Figure 1 (a), but with an $s[n]$ that contains slight noise contamination. While this contamination does not have much visual effect on $s[n]$, the fitting coefficients shown in Figure 1(d) are very different from Figure 1(b) and clearly suffer from overfitting, since the least-squares method attempts to use all degrees of freedom.

To combat this problem, this paper leverages recent developments in the field of compressed sensing and sparse reconstruction. In particular, an unstructured pruning in which the fitting method automatically selects the most appropriate subset of time shifts in $\mathbf{h}$ is used. This objective can be mathematically expressed using the $l_{1}$-norm of $\mathbf{h}$, since the $l_{1}$-norm can be used to promote a sparse solution for $\mathbf{h}$ and thus minimize the used degrees of freedom/time shifts. The reformulation of (2) thus becomes:

$$
\underset{\mathbf{h}}{\arg \min }\|\mathbf{h}\|_{1} \ni \sum_{n=0}^{N-1}\left\|s[n]-\mathbf{h}^{T} \mathbf{r}[n]\right\|_{2}<\epsilon
$$

While (3) does not have a closed-form solution, it can be solved using methods such as the Compressive Sampling Matching Pursuit (CoSaMP) [7],[8].

Given the interpretation of $h[n]$ as a filter, it follows that the elements of $h[n]$ that are encapsulated in $\mathbf{h}$ can be viewed as a model of the injury. The magnitude and indices of the non-zero elements of $\mathbf{h}$ that result from solving (3) correspond to the amplitude scales and time-shifts, respectively, of $r[n]$ that are needed to form $s[n]$. That is, $r[n]$ can be considered to originate from a single reference neural pathway, while $s[n]$ is formed from several paths (with respect to the reference path $r[n]$ ) due to the injury. Each of these paths induces a time-shift and amplitude scaling effect, the result of which, when superposed at the cortex, forms the signal $s[n]$. Therefore, mild SCI should result in a solution for $\mathbf{h}$ that is close to a unit impulse function, whereas severe SCI should cause $\mathbf{h}$ to differ greatly from a unit impulse function. As such, the impulse distance $I$ is proposed to quantify the level of injury and is defined as:

$$
I_{D}=\frac{1}{2}\left[\frac{\sum_{m=-M}^{M}|h[m]-\delta[m-k]|}{1+\sum_{m=-M}^{M}|h[m]|}+\left|\frac{k}{M}\right|\right]
$$

where $k$ is the time shift that minimizes the numerator of the first term in (4). In this manner, $I$ measures the similarity of $\mathbf{h}$ to a shifted unit impulse with a penalty for impulse locations away from $m=0$.

\section{REsults}

The baseline SEP signal $r[n]$ and SCI affected SEP signal $s[n]$ for the rodents used in this paper is shown in Figure 2. The signals in the top row of Figure 2 corresponds to the rodents that were inflicted with spinal hemitransection, while the signals in the bottom row correspond to the rodents that were subjected to the more severe full transection.

Figure 3 shows colormap plots of the elements of h obtained from solving (3) using the corresponding signals in Figure 2. It can be seen that for Rodents 1-5 

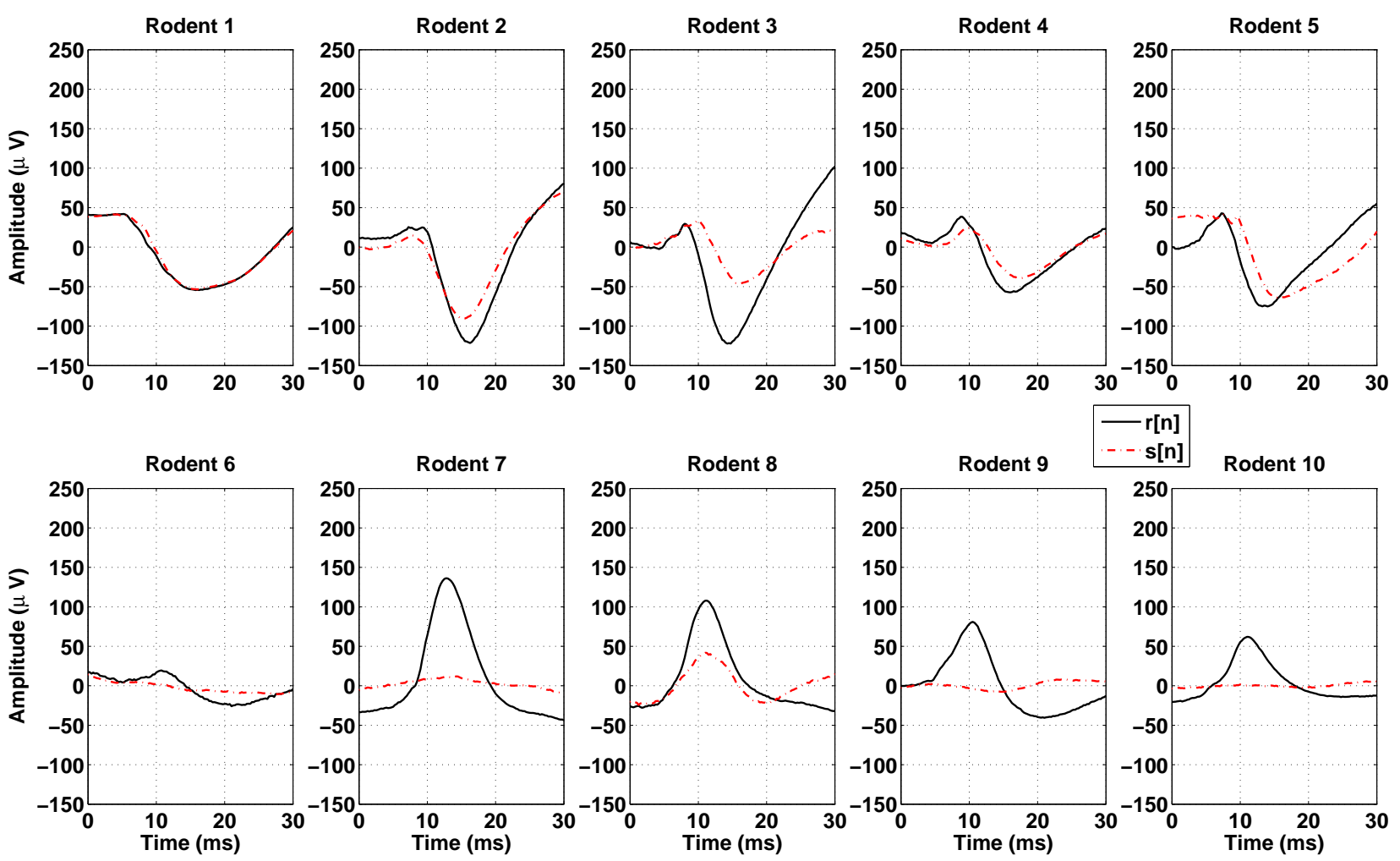

Figure 2: $r[n]$ and $s[n]$ for hemistransection (top row) and full spinal transection (bottom row).

(which have been subjected to only spinal hemitransection), the elements of $\mathbf{h}$ contain only one significant coefficient at a small or zero time shift, which means that $s[n]$ is morphologically similar to $r[n]$. This can also be verified by the corresponding SEP signals in the top row of Figure 2. Conversely, it can be seen in Figure 3 that for Rodents 6-10 (which have been subjected to severe full spinal transection), the elements of $\mathbf{h}$ contain several small-valued coefficients distributed over various time shifts, which implies that $s[n]$ is not morphologically similar to $r[n]$. This can again be verified by a visual examination of the corresponding SEP signals in the bottom row of Figure 2, and is consistent with the fact that the rodents in this case have been subjected to full spinal hemitransection.

In order to compare the proposed method with existing SCI assessments methods such as the quantitative spectral coherence measure [2] and the qualitative BBB test [5], it is necessary to condense $\mathbf{h}$ to a single value, which is accomplished through use of the impulse distance $I$ in (4). Figure 4 shows the impulse distance, spectral coherence, and BBB scores for all rodents. These values agree well with each other, as shown by their correlation values in Table 1 .
TABLE I: Correlation magnitude for various SCI assessment methods

\begin{tabular}{|l||r|r|}
\hline & $\begin{array}{r}\text { Spectral } \\
\text { Coherence }\end{array}$ & BBB Score \\
\hline \hline $\begin{array}{l}\text { Impulse Dis- } \\
\text { tance }\end{array}$ & 0.92 & 0.67 \\
\hline $\begin{array}{l}\text { Spectral Co- } \\
\text { herence }\end{array}$ & 1 & 0.71 \\
\hline
\end{tabular}

It may be noted that a quantitative description of the SEP signal morphology that relates to the level of SCI is of practical use because it lends itself to interpretation without requiring a trained examiner. Moreover, nerve injury from SCI results in demyelination that reduces both signal propagation velocity and amplitude. This temporal and amplitude change is accounted for in the proposed methodology. Compared to spectral coherence which only accounts for amplitude characteristics, the vector $\mathbf{h}$ encapsulates temporal and amplitude characteristics of $s[n]$ vis-àvis $r[n]$, and the impulse distance $I$ further consolidates $\mathbf{h}$ to a single number. The proposed methodology thus complements existing approaches and assists in 


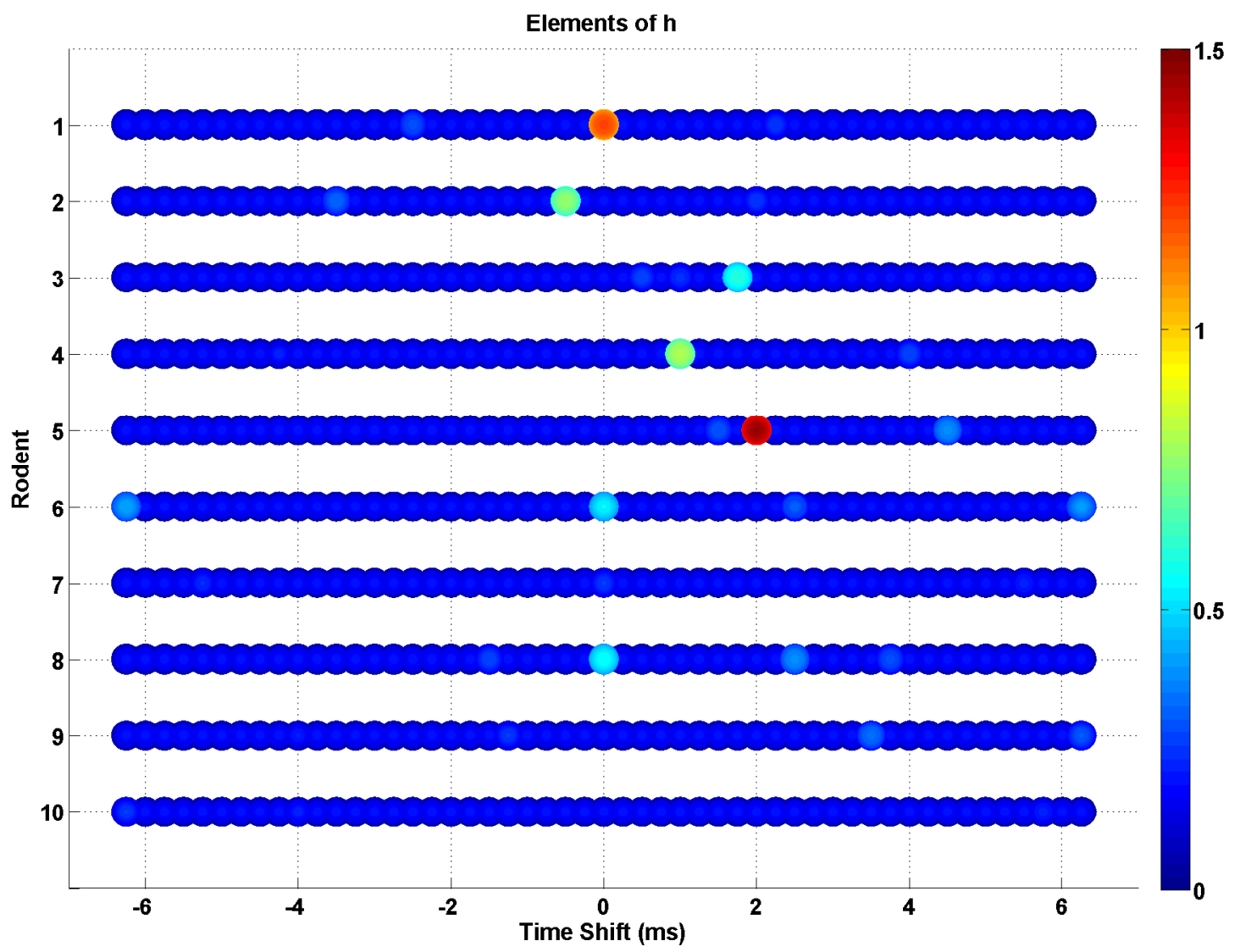

Figure 3: Elements of $\mathbf{h}$ resulting from solving (3) for the corresponding signals in Figure 2. Note that Rodents 1-5 have been subjected to hemitransection, whereas Rodents 6-10 have been subjected to full transection.

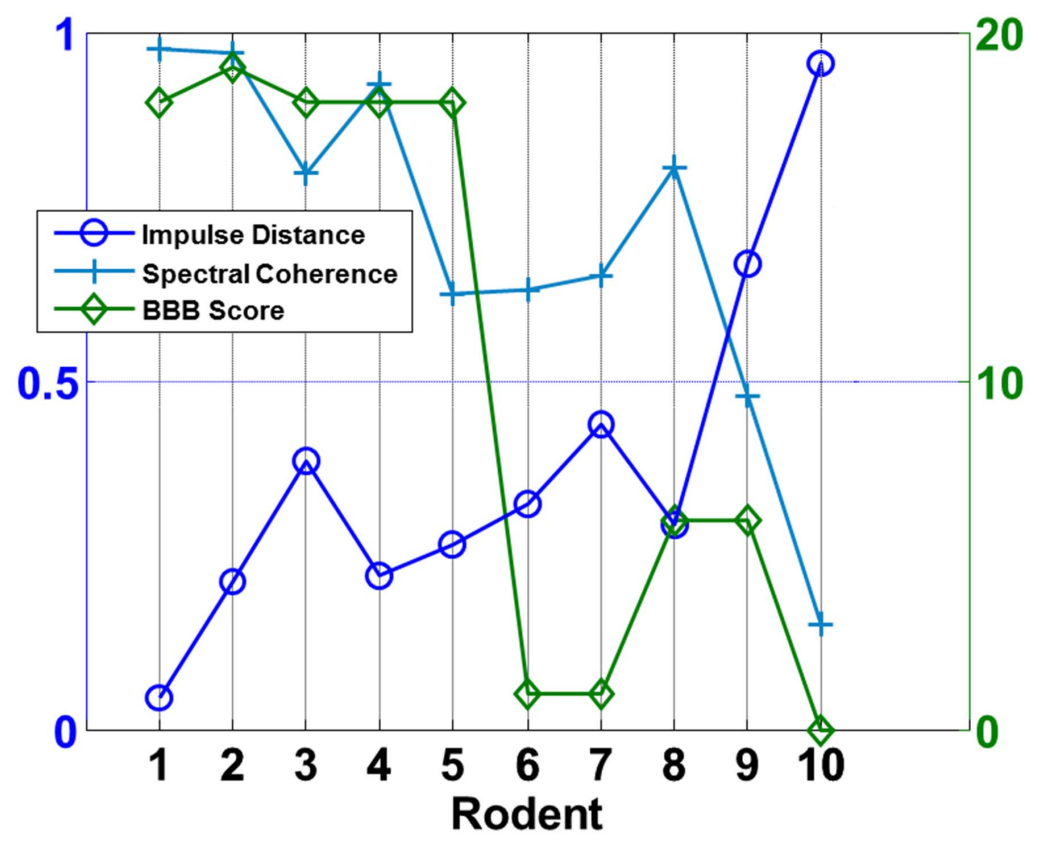

Figure 4: Comparison of Impulse Distance, Spectral Coherence, and BBB Score values for Rodents. 
furthering the understanding of the electrophysiological characteristics of SCI.

\section{CONCLUSION}

A measure was developed to encapsulate that morphological similarity between an SEP signal from a healthy spinal pathway and injured spinal pathway as a means to quantify the level of SCI. The proposed measure uses ideas from sparse representation in order to encapsulate both the time and amplitude characteristics of the injury. These characteristics were further condensed to a single number, which was shown to be consistent with existing quantitative and qualitative SCI assessment methods, while also providing a richer description and understanding of the morphological deformation of the SEP caused by SCI.

\section{REFERENCES}

[1] F. Bazley. C. Hu, BS, A. Maybhate, A. Pourmorteza, N. Pashai, N. Thakor, C. Kerr, and A. All, "Electrophysiological Evaluation of Sensory and Motor Pathways After Incomplete Unilateral Spinal Cord Contusion," Journal of Neurosurgery: Spine, vol. 16, pp. 414-423, Apr. 2012

[2] H. Al-Nashash et al, "Spinal Cord Injury Detection and Monitoring Using Spectral Coherence," IEEE Transactions on Biomedical Engineering, vol. 56, no. 8, pp. 1971-1979, Aug. 2009.

[3] H. Mir, H. Al-Nashash, D. Kerr, N. Thakor, and A. All, "Histogram Based Quantification of Spinal Cord Injury Level Using Somatosensory Evoked Potentials," Proceedings of 2010 International Conference of the IEEE Engineering in Medicine and Biology Society, pp. 4942 - 4945, Sep. 2010

[4] A. Olenko, K. Wong, H. Mir, and H. Al-Nashash "Generalized Correlation Index for Quantifying Signal Morphological Similarity," IET Electronic Letters, vol. 52, pp. 1832 - 1834, Oct. 2016

[5] D. Basso, M. Beattie, and J. Bresnahan, "A sensitive and reliable locomotor rating scale for open field testing in rats," Journal of Neurotrama, vol. 12, pp. 1-21, 1995.

[6] H. Mir, H. Al-Nashash, J. Kortelainen, and A. All, "Novel Modeling of Somatosensory Evoked Potentials for the Clinical Assessment of Spinal Cord Injury," Accepted in IEEE Transactions on Biomedical Engineering, vol. PP, no. 99, pp. 1-1.

[7] D. Needell and J. Tropp,"CoSaMP: Iterative signal recovery from incomplete and inaccurate samples," Applied and Computational Harmonic Analysis, vol. 26, no. 3, pp. 301321, May 2009.

[8] M. Davenport, D. Needell, and M. Wakin, "Signal space CoSaMP for sparse recovery with redundant dictionaries, IEEE Transactions on Information Theory, vol. 59, no. 10, pp. 68206829, Oct. 2013. 